\title{
Statistics of the System Performance in a Scrambled Recirculating Loop With PDL and PDG
}

\author{
Yu Sun, Student Member, IEEE, A. O. Lima, I. T. Lima, Jr., J. Zweck, L. Yan, C. R. Menyuk, Fellow, IEEE, and \\ G. M. Carter, Senior Member, IEEE
}

\begin{abstract}
We have demonstrated that with the loop-synchronous scrambling technique, the $Q$ distribution of a recirculating loop closely resembles that of a straight-line system. By carefully choosing the scrambling rate, we show that slow scrambling at the transmitter improves the system performance and reduces the performance variation. We investigate the system performance for different polarization-dependent loss (PDL) levels and obtain excellent agreement between the experimental and simulation results. Our results show, for the first time, that the repolarization of the noise due to significant PDL causes an asymmetric $Q$-factor distribution.
\end{abstract}

Index Terms-Optical fiber communication, optical fiber polarization, optical noise, optical receiver, polarization-dependent loss (PDL), $Q$ factor.

\section{INTRODUCTION}

$\mathbf{R}$ ECIRCULATING loops, which are widely used as experimental models for straight-line systems, do not adequately emulate the polarization effects in a straight-line system due to the periodicity of the optical path [1]-[3]. Moreover, the system performance in a loop system also depends on the input polarization state of the light and the fiber orientations, both of which drift with time, making it very difficult to repeat the statistical distribution in such a system [4]. We overcome these limitations and are able to emulate a straight-line system by using a loop-synchronous scrambler [2], which provides a random rotation for each round trip to break the periodicity of the optical path and to simulate random changes of the birefringence in straight-line systems. Moreover, the random rotations generated by the loop-synchronous scrambler are repeatable, which enable us to characterize the system performance for a given set of rotations. In modern wavelength-division-multiplexing systems, the polarization-dependent gain (PDG) in the amplifiers is effectively eliminated due to a large channel count. In order to reduce the effect of PDG in our single channel demonstration, we employed a second polarization scrambler at the transmitter of the system.

Manuscript received December 27, 2002; revised April 11, 2003. This work was supported by the National Science Foundation and by the DOE.

Y. Sun, A. O. Lima, I. T. Lima, Jr., J. Zweck, L. Yan, and C. R. Menyuk are with the Department of Computer Sciences and Electrical Engineering, University of Maryland Baltimore County, Baltimore, MD 21227 USA (e-mail: yusun@umbc.edu).

G. M. Carter is with the Department of Computer Sciences and Electrical Engineering, University of Maryland Baltimore County, Baltimore, MD 21227 USA, and also with The Laboratory for Physical Sciences, University of Maryland, College Park, MD 20740 USA.

Digital Object Identifier 10.1109/LPT.2003.814871

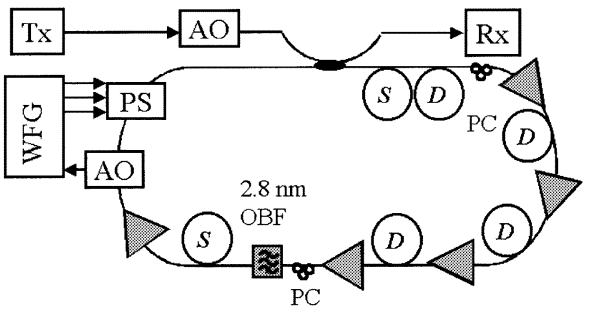

Fig. 1. Schematic system configuration. $D$ indicates the dispersion-shifted fiber and $S$ indicates the single-mode fiber.

The statistics of the signal-to-noise ratio (SNR) due to a concatenation of polarization-dependent loss (PDL) has been studied theoretically [5]. However, the relationship between the SNR and the $Q$ factor, which is often used to evaluate the system performance, is not straightforward, especially when the noise is partially polarized. In particular, the statistics of SNR do not give a complete picture of the statistics of the $Q$ factor. We investigated, theoretically and experimentally, the statistics of the $Q$ factor of a recirculating loop system with the loop-synchronous scrambler for several different PDL levels. We show that repolarization of the noise causes an asymmetric $Q$-factor distribution. We obtain excellent agreement between measured results and simulated results, which correctly accounts for the effect of partially polarized noise in the receiver.

\section{EXPERIMENTAL SETUP}

Fig. 1 shows a schematic diagram of the recirculating loop used in our experiments [6]. In this dispersion-managed soliton system, we propagate $10-\mathrm{Gb} / \mathrm{s}$ return-to-zero pulses modulated by a $2^{15}-1$ pseudorandom binary sequence pattern over a distance of $18000 \mathrm{~km}$. The loop is configured with a dispersion map with $100 \mathrm{~km}$ of dispersion-shifted fiber with $D=$ $-1 \mathrm{ps} / \mathrm{nm} \cdot \mathrm{km}$, followed by 7-km single-mode fiber with $D=$ $17 \mathrm{ps} / \mathrm{nm} \cdot \mathrm{km} . \mathrm{A} \mathrm{LiNbO}_{3}$ polarization scrambler, controlled by a programmable waveform generator (WFG), generates the random rotations for each round trip. The WFG is triggered by the driver of acoustooptic (AO) switches to insure the loop-synchronous scrambling. The scrambling rate is slower than the erbium-doped fiber amplifier (EDFA) response time. The PMD of the fiber is negligible, and the PDG is $0.05 \mathrm{~dB}$ per EDFA.

\section{SimUlation MODEL}

In the simulations, we used a reduced Stokes model [7] to follow the state of polarization (SOP) of the signal and the noise during propagation. In the receiver, to correctly account for the repolarization of the noise, we computed the $Q$ factor from the 
SNR of the electrical current using the newly developed formula [8]

$$
Q=\frac{\mathrm{SNR}}{1+\sqrt{1+2 \Gamma_{S-\mathrm{ASE}} \Gamma \kappa \mathrm{SNR}}} \sqrt{\Gamma \mu} .
$$

In this formula, the parameters $\Gamma$ and $\Gamma_{S \text {-ASE }}$ are given by

$$
\Gamma=\frac{1}{1+\mathrm{DOP}_{n}^{2}} \quad \text { and } \quad \Gamma_{S-\mathrm{ASE}}=\frac{1}{2}\left(1+\mathrm{DOP}_{n} \hat{\mathbf{s}}_{s} \cdot \hat{\mathbf{s}}_{\mathbf{n}}\right)
$$

where the unit-length Stokes vectors $\hat{\mathbf{s}}_{s}$ and $\hat{\mathbf{s}}_{n}$ give the direction of the Stokes vectors of the signal and the noise, and $\mathrm{DOP}_{n}$ is the degree of polarization of the noise defined as the power ratio of the polarized part of the noise to the total noise. The parameter $\Gamma$ gives the ratio of the variances due to noise-noise beating in the receiver for unpolarized noise to that for arbitrarily polarized noise. The parameter $\Gamma_{S \text {-ASE }}$ gives the portion of the noise that beats with the signal. When the noise is unpolarized, $\Gamma=1$ and $\Gamma_{S \text {-ASE }}=0.5$. When the noise is co-polarized with the signal, $\Gamma=0.5$, and $\Gamma_{S \text {-ASE }}=1$. The parameters $\kappa$ and $\mu$, which are independent of the polarization states of the signal and noise, depend on the pulse format and the shapes of the optical and electrical filters [9]. For the receiver that we used in experiments, $\kappa=1.7$ and $\mu=38.6$.

\section{RESULTS AND DISCUSSIONS}

We compare the system performance with and without the loop-synchronous scrambler by measuring the $Q$-factor distributions in the loop. In the experiments, we obtained the $Q$ factor from the margin measurements of the bit-error rate (BER) [10]. In order to focus on the behavior of the optical noise, we carefully subtract the electrical noise background, obtained from the back-to-back experiment, from the data measured at $10000 \mathrm{~km}$. We also employ a second polarization scrambler in the transmitter to eliminate the effect of PDG.

Although the optical path of an uncontrolled loop system is quasi-static, it changes from day to day. This changes not only the random realization of the fiber birefringence but also the accumulated PDL of the system. To fix the PDL level, we consider one round trip of the loop from the first EDFA to the AO-switch as a black box that generates PDL. By adjusting the polarization controllers between the PDL elements in the black box, we vary the PDL per round trip from 0.13 to $0.6 \mathrm{~dB}$. Once the PDL level is chosen, we control the SOP of the light within the loop by adjusting the polarization controller outside the black box and monitor the accumulated PDL, using the build-up of the DOP of the noise [3] regularly to avoid any drift in the round-trip PDL.

We first measured the $Q$ distribution of the loop system at $10000 \mathrm{~km}$ using 400 samples when the PDL per round trip was $0.2 \mathrm{~dB}$, as described in [3]. To simulate the steady nature of the loop system, the loop-synchronous scrambler provided the same rotations for each round trip in one $Q$-factor measurement. The rotations are chosen randomly for different $Q$-factor samples. In Fig. 2, we show the measured $Q$ distribution without loop-synchronous scrambling as a histogram. Because of the periodicity, the unscrambled loop system has a much broader $Q$-factor distribution than we would expect for the corresponding straight-line system. We also show the cor-

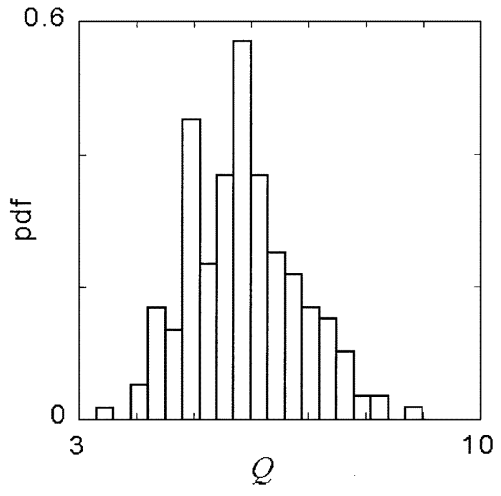

Fig. 2. Histogram of the measured $Q$-factor distribution of the recirculating loop when the PDL is $0.2 \mathrm{~dB}$ per round trip.

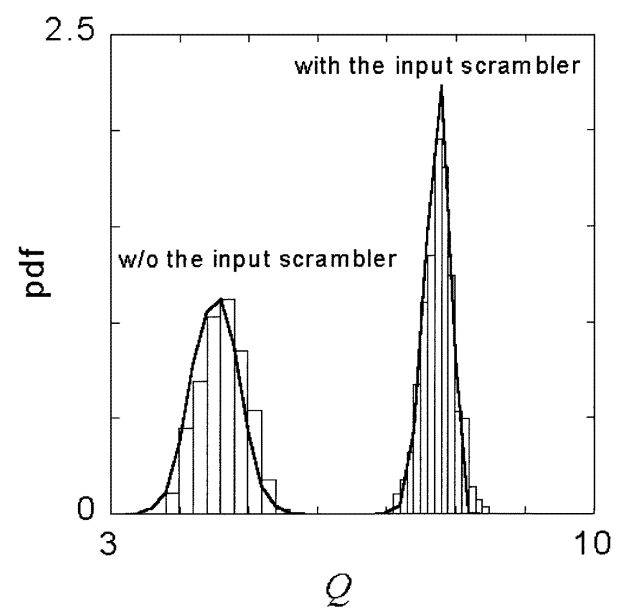

Fig. 3. Comparison of the measured and the simulated $Q$-factor distributions when the PDL is $0.2 \mathrm{~dB}$ per round trip with the loop-synchronous scrambler, with and without the transmitter polarization scrambler. We show the measured $Q$-factor distributions as histograms and the corresponding simulated results as solid lines.

responding measured result with loop-synchronous scrambling as a histogram in Fig. 3. The solid line is the simulated result. The loop-synchronous scrambler generated a different random rotation for each round trip. For each $Q$ sample, an independent sequence of random rotations was repeated until the measurement of the $Q$ factor was completed. This loop-synchronous scrambling technique breaks the periodicity of the optical path and accurately emulates the statistics of PMD and PDL of a straight-line system. Although the $Q$ distribution tends to be aligned with the low $Q$ portion of the $Q$ distribution shown in Fig. 2 due to the reduction of SNR, the $Q$-factor distribution is much narrower and closely resembles the $Q$ distribution for a straight-line system. The measured and simulated results are in excellent agreement.

We also employed a polarization scrambler at the transmitter to reduce the effect of PDG. In our experiment, we carefully chose a slow scrambling rate of $12.6 \mathrm{kHz}$ to reduce both effects of PDG and the amplitude modulation induced by PDL [11]. In Fig. 3, we show the measured and simulated results for the system with both transmitter and loop-synchronous scramblers. With the transmitter scrambler, in addition to improving the average performance of the system, we also further decrease the width of the $Q$-factor distribution. 


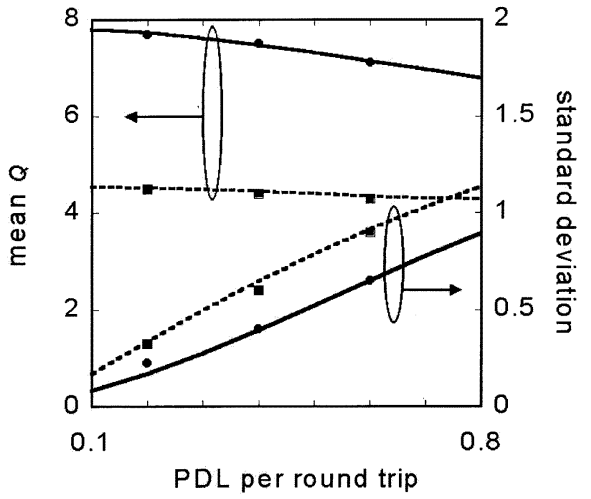

Fig. 4. Comparison of the measured and the simulated mean and standard deviations of $Q$-factor distributions of a scrambled loop system with different PDL levels. The solid lines and the dashed lines represent simulated results with and without the input polarization scrambler. The filled circles and squares are the measured results for the system with and without the input polarization scrambler, respectively.

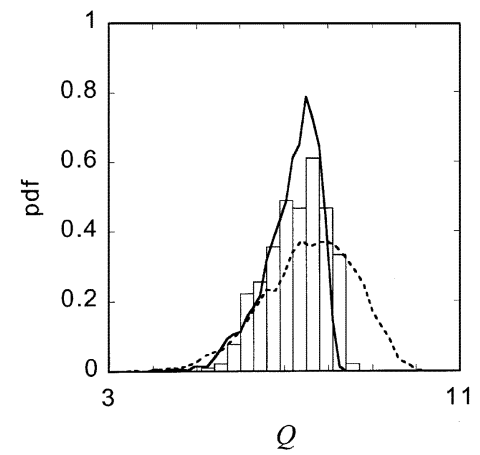

Fig. 5. Comparison of the measured and the simulated $Q$-factor distributions of a scrambled loop with the input polarization scrambler when the PDL is $0.6 \mathrm{~dB}$ per round trip. We show the measured $Q$-factor distribution as histograms and the corresponding simulated result as a solid line. The dashed line is a simulated result, assuming the noise prior to the receiver is unpolarized.

We measured the $Q$-factor distributions of the scrambled loop system at $10000 \mathrm{~km}$ with and without the input scrambler with $\mathrm{PDL}=0.2 \mathrm{~dB}, \mathrm{PDL}=0.4 \mathrm{~dB}$, and $\mathrm{PDL}=0.6 \mathrm{~dB}$ per round trip. The comparison of the simulated and measured means and standard deviations of these $Q$ distribution that we show in Fig. 4 demonstrates that large PDLs in the system tends to cause significant variation of the system performance, while producing a small degradation in the average system performance.

Furthermore, with the transmitter scrambler, for a large PDL such as $\mathrm{PDL}=0.6 \mathrm{~dB}$ per round trip, the $Q$ distribution is asymmetric, as shown in Fig. 5. Our simulation shows that it is the repolarization of the noise that causes the asymmetry of the $Q$ distribution. Since the transmitter scrambler eliminates the effect of PDG, the noise tends to repolarize. For those samples where the signal is often closely aligned with the low-loss axis of PDL elements, the SNR is larger than the average SNR. In this case, the noise tends to become co-polarized with the signal because the noise perpendicular to the signal is reduced by the high-loss axis of PDL elements. Therefore, the signal-noise beating is enhanced and the $Q$ factor is lower than that of the system with the same SNR but where the noise is unpolarized. Consequently, the large $Q$ portion of the $Q$ distribution with unpolarized noise is missing for polarized noise, and the $Q$ distribution is asymmetric. By contrast, if in the simulation we artificially assume that the noise prior to the receiver is unpolarized by setting $\Gamma=1$ and $\Gamma_{S \text {-ASE }}=0.5$ and keeping all the other parameters the same, then, as we show with a dashed line in Fig. 5, the $Q$ distribution is much more symmetric. Our results show that to truly predict the system performance and estimate the outage probability, one must include effects of partially polarized noise in the receiver model.

\section{CONCLUSION}

We demonstrate that with the loop-synchronous scrambling technique, the $Q$ distribution of a recirculating loop closely resembles that of a straight-line system. By carefully choosing the scrambling rate, we show that slow scrambling at the transmitter improves the system performance and reduces the performance variation. We investigate the system performance for different PDL levels and obtain excellent agreement between the experimental and simulation results. Our results show that when significant PDL in the system repolarizes the noise, the $Q$-factor distribution is asymmetric. To accurately predict the system performance and estimate the outage probability, one must take into account effects of partially polarized noise in the receiver model.

\section{REFERENCES}

[1] Y. Sun, B. S. Marks, I. T. Lima, Jr., K. Allen, G. C. Carter, and C. R. Menyuk, "Polarization state evolution in recirculating loops," in Tech. Dig. OFC 2002, Paper ThI4.

[2] S. Lee, Q. Yu, L.-S. Yan, Y. Xie, O. H. Adamczyk, and A. E. Willner, "A short recirculating fiber loop testbed with accurate reproduction of Maxwellian PMD statistics," in Tech. Dig. OFC 2001, Paper WT2.

[3] Y. Sun, I. T. Lima, Jr., H. Jiao, J. Wen, H. Xu, H. Erifej, G. M. Carter, and C. R. Menyuk, "Study of system performance in a 107-km dispersionmanaged recirculating loop due to polarization effects," IEEE Photon. Technol. Lett., vol. 13, pp. 966-968, Sept. 2001.

[4] G. M. Carter and G. M. Yu Sun, "Making the $Q$ distribution in a recirculating loop resemble a straight line distribution," in Tech. Dig. OFC 2002, Paper ThQ5.

[5] M. Yu, C. Kan, M. Lewis, and A. Sizmann, "Statistics of signal-to-noise ratio and path-accumulated power due to concatenation of polarizationdependent loss," IEEE Photon. Technol. Lett., vol. 14, pp. 1418-1420, Oct. 2002.

[6] J. M. Jacob and G. M. Carter, "Error-free transmission of dispersion-managed solitons at $10 \mathrm{Gbit} / \mathrm{s}$ over $24500 \mathrm{~km}$ without frequency sliding," Electron. Lett., vol. 33, pp. 1128-1129, 1997.

[7] D. Wang and C. R. Menyuk, "Reduced model of the evolution of the polarization states in wavelength-division-multiplexed channels," Opt. Lett., vol. 23, pp. 1677-1679, 1998.

[8] Y. Sun, I. T. Lima, Jr., A. O. Lima, H. Jiao, J. Zweck, C. R. Menyuk, and G. M. Carter, "Receiver effects due to partially polarized noise," in Tech. Dig. OFC 2003, to be published.

[9] I. T. Lima, Jr., A. O. Lima, J. Zweck, and C. R. Menyuk, "Computation of the $Q$-factor in optical fiber systems using an accurate receiver model," in Tech. Dig. OFC 2003, to be published.

[10] N. S. Bergano, F. W. Kerfoot, and C. R. Davidsion, "Margin measurements in optical amplifier system," IEEE Photon. Technol. Lett., vol. 5, pp. 304-306, Mar. 1993.

[11] M. G. Taylor, "Improvement in $Q$ with low frequency polarization modulation on transoceanic EDFA link," IEEE Photon. Technol. Lett., vol. 6, pp. 860-862, July 1994. 